\section{ORIGINAL RESEARCH}

\section{P.G. Sämann \\ M. Knop \\ E. Golgor \\ S. Messler \\ M. Czisch \\ F. Weber}

\title{
Brain Volume and Diffusion Markers as Predictors of Disability and Short-Term Disease Evolution in Multiple Sclerosis
}

BACKGROUND AND PURPOSE: MRI markers of neuroaxonal damage in MS have emerged as critical long-term predictors of MS-related disability. Here we investigated the potential of whole-brain diffusivity and brain volume for the prediction of cross-sectional disability and short- to medium-term clinical evolution.

\begin{abstract}
MATERIALS AND METHODS: In this multimodal prospective longitudinal MRI study of 54 patients with MS (87\% under immunomodulatory therapy, baseline and follow-up at a median of 12 months), ADC histogram analysis, WM lesion load, BPF, whole-brain atrophy rate, MSFC score, and EDSS score were obtained. A total of 44 patients with no relapse at both time points were included.
\end{abstract}

RESULTS: At both time points, ADC histogram analysis provided robust predictors of the MSFC scores (maximal $R^{2}=0.576, P<.001$ ), incorporated cognition and fine-motor skill subscores, and EDSS scores. Significant changes beyond physiologic age-related changes at follow-up were noted for ADC histogram markers and BPF. Stronger diffusivity alterations and brain volume at baseline predicted MSFC decline, as demonstrated by multiple linear regression analysis (mean ADC, $R^{2}=0.203 ; P=$ $.003)$ and lower baseline BPF in patients with declined compared with stable MSFC scores $(P=.001)$. Results were independent of intercurrent relapses.

CONCLUSIONS: Diffusion histogram analysis provided stable surrogates of disability in MS and proved sensitive for monitoring disease progression during a median of 12 months. Advanced neuroaxonal pathology at baseline was indicative of an increased risk for sustained progression during a median of 12 months, independent of intercurrent relapses.

ABBREVIATIONS: BPF = brain parenchyma fraction; $\mathrm{Cl}=$ confidence interval; EDSS = Expanded Disability Status Scale; GM = gray matter; MSFC = MS Functional Composite; 9-HPT = 9-Hole Peg Test; PASAT = Paced Auditory Serial Addition Test; PBVC = percentage brain volume change; TWT $=$ timed walk test; $W_{M L L}$ perc $=W M$ lesion load volume as percentage of total WM volume

A cute inflammation and demyelination, secondary neuroaxonal pathology, and additional neurotrophic disturbances conjointly lead to clinical impairment in MS. ${ }^{1,2}$ Among these factors, the cumulative neuroaxonal damage is a particularly strong determinant of disability. ${ }^{3}$ Exceeding a certain threshold of neuroaxonal damage might accelerate a patient's transition to secondary-progressive MS or "sustained progression." 3 Therefore, MRI techniques that are sensitive to the cumulative neuroaxonal damage such as volumetry ${ }^{2,4}$ and $\mathrm{DWI}^{5}$ warrant further investigation to improve the clinical management of MS.

Brain-volume loss in MS is a multifactorial process that originates from inflammatory focal axonal damage and depletion of myelin sheaths, secondary neuroaxonal degenera-

\section{Received July 12, 2011; accepted after revision October 16}

From the Neuroimaging Research Group (P.G.S., E.G., M.C.) and Inflammatory Disorders of the Central Nervous System Research Group (M.K., F.W.), Max Planck Institute of Psychiatry, Munich, Germany; and Department of Statistics (S.M.), Ludwig-Maximilians-Universität, Munich, Germany. Dr Golgor is currently affiliated with the Institute of Diagnostic Radiology, University Hospital Carl Gustav Carus, Technical University Dresden, Dresden, Germany. Dr Messler is currently affiliated with INC Research GmbH, Munich, Germany. The authors declare no competing interests.

Please address correspondence to P.G. Sämann, MD, Kraepelinstr 2-10, 80804 Munich, Germany; e-mail: saemann@mpipsykl.mpg.de

Indicates article with on-line appendix

Indicates article with supplemental on-line figure.

http://dx.doi.org/10.3174/ajnr.A2972 tion, and other immunologically triggered neurotrophic disturbances. ${ }^{2,4}$ It can be observed across all MS subtypes, ${ }^{6}$ even in early stages, ${ }^{7}$ and its relation to physical and cognitive disability is generally recognized..$^{8-10}$ In relapsing-remitting MS, between $47 \%$ and $81 \%$ of brain atrophy was ascribed to the previous cumulative gadolinium enhancement. ${ }^{11}$ Other studies suggest that brain atrophy is a consequence of diffuse pathology rather than focal lesions. ${ }^{4,12}$ In fact, signs of strong tissue destruction may occur during the course of MS despite low cumulative inflammatory activity. ${ }^{13}$ The strong clinical relevance of brain volumetry in MS is supported by correlations between baseline brain volume and disability occurring 8 years later ${ }^{14}$ and associations between early brain atrophy rates and clinical deterioration. ${ }^{15}$

DWI detects alterations of microscopic diffusion processes in MS due to a variety of factors, including loss of myelin sheaths, loss of axonal membranes, neuronal apoptosis, and gliosis formation. ${ }^{5}$ It is now well-established that diffusivity measurements are sensitive to MS-related pathology in brain areas that appear normal on conventional T2- and T1weighted images. ${ }^{5,16-18}$ Notably, diffusivity changes parallel grades of axonal pathology in animal models ${ }^{19}$ and in humans, ${ }^{20,21}$ which might explain correlations of diffusivity markers with patient disability status. ${ }^{22-24}$ Serial application of DWI revealed progressive microstructural GM changes in untreated relapsing-remitting $\mathrm{MS},{ }^{25}$ and a good prediction 


\begin{tabular}{|c|c|c|}
\hline & Patients & Controls \\
\hline No. & 44 & 54 \\
\hline Age (mean \pm SD) (yr) & $37.4(9.6)$ & $38.2(9.1)$ \\
\hline Men/women & $16 / 28$ & $19 / 35$ \\
\hline Age of onset (mean \pm SD) (yr) & $30.2(10.1)$ & N/A \\
\hline Disease duration at baseline (mean \pm SD) (yr) & $6.6(6.3)$ & $\mathrm{N} / \mathrm{A}$ \\
\hline Disease type (RRMS/SPMS/PPMS) & $38 / 4 / 2$ & $\mathrm{~N} / \mathrm{A}$ \\
\hline Immunomodulatory therapy (o.) (\%) & & $\mathrm{N} / \mathrm{A}$ \\
\hline $0=$ No therapy $^{\mathrm{b}}$ & $7(15.9)$ & \\
\hline $1=\beta$-interferon ${ }^{\mathrm{c}}$ & $21(47.7)$ & \\
\hline $2=$ Glatirameracetate & $11(25.0)$ & \\
\hline $3=$ Immunoglobulins & $4(9.0)$ & \\
\hline $4=3$-month methylprednisolone & $1(2.2)$ & \\
\hline
\end{tabular}

Note:-NA indicates not applicable; PPMS, primary-progressive MS; RRMS, relapsingremitting MS; SPMS, secondary-progressive MS.

${ }^{a}$ No significant differences between patients with RRMS and SPMS/PPMS were detected for age, age of onset, and disease duration.

${ }^{b}$ One patient changed to interferon treatment during the study period.

${ }^{c}$ Three patients changed from interferon to mitoxantrone therapy during the study period.

of the potential for the clinical status after 5 years in primary-progressive $\mathrm{MS}^{26}$

Despite the sensitivity of DWI, however, there are scant serial data on longitudinal DWI measures, particularly in treated relapsing-remitting MS and in combination with sensitive clinical monitoring instruments such as the MSFC score. ${ }^{27}$ Furthermore, serial studies have either focused on $\mathrm{DWI}^{25,26,28,29}$ or brain volume measurements, ${ }^{6,9,11,14,30-36}$ with only 1 serial study on primary-progressive MS and secondary-progressive MS using both techniques. ${ }^{37}$ In this prospective, longitudinal, and multimodal MRI study on patients with MS under treatment, we investigated the potential of whole-brain diffusivity and brain volume for the prediction of cross-sectional disability and short-to-medium-term clinical evolution.

\section{Materials and Methods}

\section{Patients with MS, Clinical Evaluation, and Controls}

Patients were consecutively recruited from the outpatient clinic and the neurologic ward of the Max Planck Institute of Psychiatry, Munich. They fulfilled the criteria of definite MS according to McDonald et $\mathrm{al}^{38}$ with the major proportion classified as relapsing-remitting MS (47 patients) (secondary-progressive MS, 5 patients; primaryprogressive MS, 2 patients). Patients with relapses at baseline or at follow-up $(n=10)$ were excluded from clinicoradiologic correlation analysis to avoid confounding influences from transient clinical exacerbation, ${ }^{39}$ leaving 44 patients for the final analysis (Table 1). Disease duration was estimated from a detailed clinical history and file review. Thirty-seven of 44 patients ( $84 \%$ ) received immunomodulatory therapy at study entry (Table 1). At baseline and follow-up after a median of 12 months (median, 371 days; range 308-702 days), the EDSS ${ }^{40}$ and MSFC scores, ${ }^{27}$ comprising the TWT, 9-HPT, and a 3 -second version of the PASAT, were obtained. Patients with $\geq 1$ relapse during the observation interval were identified for post hoc analyses as specified below. The number of patients with MSFC scores available at both time points varied between 38 and 40 due to (disease-related) dropouts in subtests.

Follow-up MSFC scores were interpolated to a 12-month interval (annualized scores). Clinical progression was parameterized as the difference between baseline and annualized follow-up scores. For the
MSFC sum score and subscores, patients with a negative annual change value were assigned to the respective progression group in a first step. Second, to reduce false classification into the MSFCprogression group, we classified $20 \%$ of the patients with the lowest progression rates as stable. For the EDSS, an increase of $\geq 0.5$ point between baseline and follow-up with confirmation 3 months later was classified as EDSS progression.

For proof-of-concept comparisons and estimation of age effect, an age and sex-matched control group free of neurologic or psychiatric disease underwent the same MRI protocol once $(n=54$, Table $1)$.

The study followed the principles of the Declaration of Helsinki and was approved by the local ethics committee. All participants gave their written informed consent.

\section{MR Imaging Acquisition and Postprocessing: Overview}

Images were acquired on a clinical $1.5 \mathrm{~T}$ scanner (Signa Excite; GE Healthcare, Milwaukee, Wisconsin). Sequence details and postprocessing steps are described in the on-line supplemental material. In brief, we extracted the following MRI markers: 1) Whole-brain ADC histograms were calculated; mean, variance, skew, and peak-height values were extracted. ${ }^{18,41}$ 2) The BPF (brain parenchyma volume divided by total intracranial volume ${ }^{42}$ ) was calculated at baseline and follow-up from T2-weighted images with high in-plane resolution and CSF/parenchyma contrast by using. 3) The brain-volume change between baseline and follow-up (PBVC) was calculated by using the SIENA algorithm of the FSL software (http://www.fmrib.ox.ac.uk, version 3.2).$^{43}$ 4) For WM lesion load quantification, multispectral image segmentation based on an expectation maximization algorithm was used. ${ }^{44}$ 5) Axial and coronal postgadolinium images of both time points were screened by 2 raters (F.W., P.G.S.) blinded to patient identity and time points.

\section{Statistical Analysis}

Baseline MRI measures of all patients were compared with those of controls by using univariate multivariate analysis of covariance based on Wilks $\lambda$, covarying for age. Group $\times$ age interaction effects were explored, and the term was removed from the model if not significant $(P>.05)$.

Follow-up MRI variables (ADC histogram metrics, BPF, and $\mathrm{WMLL}_{\text {perc }}$ ) as well as follow-up clinical scores were interpolated to a 12-month interval. Paired tests were used to compare baseline and annualized MRI and parametric clinical markers (Wilcoxon signed rank test for EDSS; $t$ test for other variables). The patients' annual atrophy rates as calculated by the SIENA algorithm were compared against zero by using a 1 -sample $t$ test. Annual change rates and $95 \%$ CIs for MRI variables of the control group were estimated by linear regression analysis.

For cross-sectional clinico-radiologic correlations, the Spearman rank correlation tests (for EDSS) and the Pearson partial correlation tests corrected for age (for MSFC scores) were applied to baseline and follow-up values. For the 28 MSFC- and 7 EDSS-related tests, Bonferroni-adjusted significance thresholds were defined (0.05/28 0.0018 and $0.05 / 7 \sim 0.0071$, respectively) to adjust for explorative testing. For baseline and follow-up MSFC and EDSS, stepwise linear regression analysis (variable entry at $P<.05$, variable removal at $P>$ .10) was appended to identify independent predictors among the MRI variables. Reported $R^{2}$ values represent the proportion of explained variance, adjusted for the entry of multiple regressors.

Prediction of clinical progression was analyzed in 2 ways: 1) Base- 


\begin{tabular}{|c|c|c|c|c|c|c|c|}
\hline Score & No. & Baseline & Follow-Up ${ }^{a}$ & $P$ Value $^{\mathrm{b}}$ & Split Threshold $^{\mathrm{c}}$ & Progression & Nonprogression \\
\hline EDSS (median) (range) & 44 & $2.0(0-5.5)$ & $2.0(0-5.5)$ & 0.950 & $\geq 0.5^{c}$ & 9 & 35 \\
\hline MSFC (mean) (SD) & 38 & $0.550(0.415)$ & $0.549(0.475)$ & 0.969 & $<-0.047$ & 13 & 25 \\
\hline TWT (mean) (SD) ${ }^{d}$ & 39 & $-0.431(0.082)$ & $-0.412(0.098)$ & 0.105 & $>0.013$ & 16 & 23 \\
\hline 9-HPT (mean) (SD) & 40 & $0.780(0.735)$ & $0.736(0.837)$ & 0.382 & $<-0.113$ & 15 & 25 \\
\hline PASAT (mean) ${ }^{\dagger}$ & 39 & $0.460(0.693)$ & $0.531(0.800)$ & 0.414 & $<-0.101$ & 8 & 31 \\
\hline
\end{tabular}

a Annualized values for MSFC, raw values for EDSS (for details see "Materials and Methods").

b Two-sided Wilcoxon signed rank test (EDSS) and paired Student $t$ test (MSFC, annualized follow-up values), respectively.

"See "Materials and Methods" section for details on threshold definition.

d TWT.

e 9 -HPT

${ }^{\text {PASAT. }}$

Table 3: MRI markers at baseline and follow-up of all patients with MS and estimated yearly changes of control subjects

\begin{tabular}{|c|c|c|c|c|c|c|}
\hline \multirow[b]{2}{*}{ MRI Marker } & \multicolumn{4}{|c|}{ Patients } & \multirow{2}{*}{\multicolumn{2}{|c|}{$\begin{array}{c}\text { Healthy Controls } \\
\text { Estimated } \\
\text { Annual Change }\end{array}$}} \\
\hline & $\begin{array}{l}\text { Baseline } \\
\text { (Mean) }\end{array}$ & $\begin{array}{l}\text { Follow-Up } \\
\text { (Mean) }^{\mathrm{a}}\end{array}$ & $\begin{array}{c}\text { Annual Change } \\
\text { (Mean) }\end{array}$ & $P$ Value & & \\
\hline \multicolumn{5}{|l|}{$\overline{\mathrm{ADC}}$ histogram markers } & $\mathrm{B}^{\mathrm{b}}$ & $95 \% \mathrm{Cl}$ \\
\hline Mean $\left(10^{-6} \mathrm{~m}^{2} / \mathrm{s}\right)$ & $0.961 \pm 0.031$ & $0.965 \pm 0.031$ & $0.004 \pm 0.013$ & $.035^{\mathrm{c}}$ & $0.0009^{d}$ & $0.0001 ; 0.0016$ \\
\hline Variance $\left(10^{-6} \mathrm{~m}^{2} / \mathrm{s}\right)$ & $0.088 \pm 0.008$ & $0.089 \pm 0.007$ & $0.001 \pm 0.004$ & $.099^{c}$ & $0.0002^{\mathrm{d}}$ & $0 ; 0.0005$ \\
\hline Skew & $1.023 \pm 0.159$ & $0.990 \pm 0.151$ & $-0.033 \pm 0.078$ & $.008^{\mathrm{c}}$ & $-0.0038^{d}$ & $-0.0075 ;-0.0002$ \\
\hline Peak height $\left(10^{-3}\right)$ & $2.644 \pm 0.266$ & $2.599 \pm 0.253$ & $-0.045 \pm 0.144$ & $.043^{\mathrm{c}}$ & -0.0028 & $-0.0101 ; 0.0046$ \\
\hline Peak position $\left(10^{-6} \mathrm{~m}^{2} / \mathrm{s}\right)$ & $0.763 \pm 0.021$ & $0.765 \pm 0.020$ & $0.003 \pm 0.013$ & $.172^{\mathrm{c}}$ & 0.0004 & $0.0001 ; 0.0010$ \\
\hline BPF & $0.815 \pm 0.043$ & $0.811 \pm 0.039$ & $-0.004 \pm 0.016$ & $.087^{c}$ & $-0.0023^{d}$ & $-0.0033 ; 0.0013$ \\
\hline PBVC (\%) & & & $-0.464 \pm 1.233$ & $.017^{\mathrm{e}}$ & \multicolumn{2}{|c|}{ N/A } \\
\hline WMLL $_{\text {perc }}(\%)$ & $3.800 \pm 2.117$ & $3.921 \pm 2.346$ & $0.121 \pm 0.557$ & $.157^{c}$ & \multicolumn{2}{|c|}{$\mathrm{N} / \mathrm{A}$} \\
\hline Patients with enhancing lesions (\%) & 18.0 & 20.4 & $\mathrm{~N} / \mathrm{A}$ & $1.000^{f}$ & \multicolumn{2}{|c|}{$\mathrm{N} / \mathrm{A}$} \\
\hline
\end{tabular}

Note:-N/A indicates not applicable.

a Annualized values are given except for assessment of gadolinium enhancement.

${ }^{\mathrm{b}}$ Unstandardized coefficient of linear regression.

${ }^{\mathrm{c}}$ Two-sided paired $t$ test.

d Significant linear correlation with age in the control group $(P \leq .05)$

e PBVC as estimated by the SIENA method. One-sample $t$ test for comparison against zero.

${ }^{\dagger}$ Fisher exact test.

line MRI markers were compared between patients with and without MSFC progression by using analysis of covariance (2-level group factor, age as a covariate). The analysis was repeated for patients stratified according to EDSS progression. 2) Stepwise linear regression, by using the annual change of MSFC sum score and subscores as dependent variables and baseline MRI variables (ADC histogram markers, $\mathrm{BPF}, \mathrm{WMLL}_{\text {perc }}$ ) as predictor variables, was applied. To exclude any influence of relapses during the observation interval, we performed the following post hoc analyses: 1) The proportion of patients with intercurrent relapses was compared between the MSFC progression and nonprogression groups (Fisher exact test). 2) MSFC prediction analyses were repeated after exclusion of patients with intercurrent relapses and for patients with relapsing-remitting MS only.

\section{Results}

\section{Clinical Characteristics and Disease Progression}

Clinical and demographic sample information is given in Table 1. Table 2 shows comparisons of clinical baseline and annualized follow-up scores. Subtle yet nonsignificant progression of the MSFC sum score, TWT, and 9-HPT and improvement of the PASAT were noted. Twenty percent of patients showed a confirmed EDSS increase of $\geq 0.5$ points. The MSFC threshold that stratified patients into MSFCprogression and MSFC-nonprogression groups was -0.047 (13 progressive, 25 nonprogressive patients).
Patient/Control Comparison of ADC Histograms and BPF Significant differences between patients and controls were detected for ADC histogram markers (multivariate analysis of covariance, $F=13.895, P<10^{-6}$ ) with an age covariate effect $(F=4.840, P=.001)$. Similarly, mean BPF differed significantly between patients $(0.815 \pm 0.043)$ and controls $(0.837 \pm$ 0.041 ) (analysis of covariance, $F=6.592, P=.004$ ) with a covariate effect of age $(F=12.580, P=.001)$ and a trend group $\times$ age interaction $(F=3.667, P=.059)$ (On-line Table $1)$. BPF correlated with age in the control group $\left(r^{2}=0.269\right.$, $\left.P<10^{-4}\right)$ but not in the patient group $\left(r^{2}=0.024, P=.314\right)$.

\section{Longitudinal Changes of MRI Markers}

Changes were most explicit for ADC histogram skew, followed by mean ADC and peak height (Table 3 ). Mean BPF decreased by $0.42 \%(P=.087)$, with a similar yet significant mean change calculated by SIENA (PBVC, $-0.46 \% ; P=.017$ ), confirming that SIENA is more sensitive to change compared with subtraction of $2 \mathrm{BPF}$ measurements. ${ }^{43} \mathrm{WMLL}_{\text {perc }}$ did not change significantly. Annual change rates of all markers in the patient group were outside the $95 \%$ CI of the estimated coefficients of controls and exceeded control values by factors 1.7 (BPF) to 8.7 (ADC histogram skew). Gadolinium enhancement was found in 8 patients $(18.2 \%$; median gadoliniumenhancement score, 1.0 [range, 1-2]) at baseline and in 9 patients (20.4\%; median gadolinium-enhancement score, 2.0 [range, 1-10]) at follow-up. At both baseline and follow-up, 


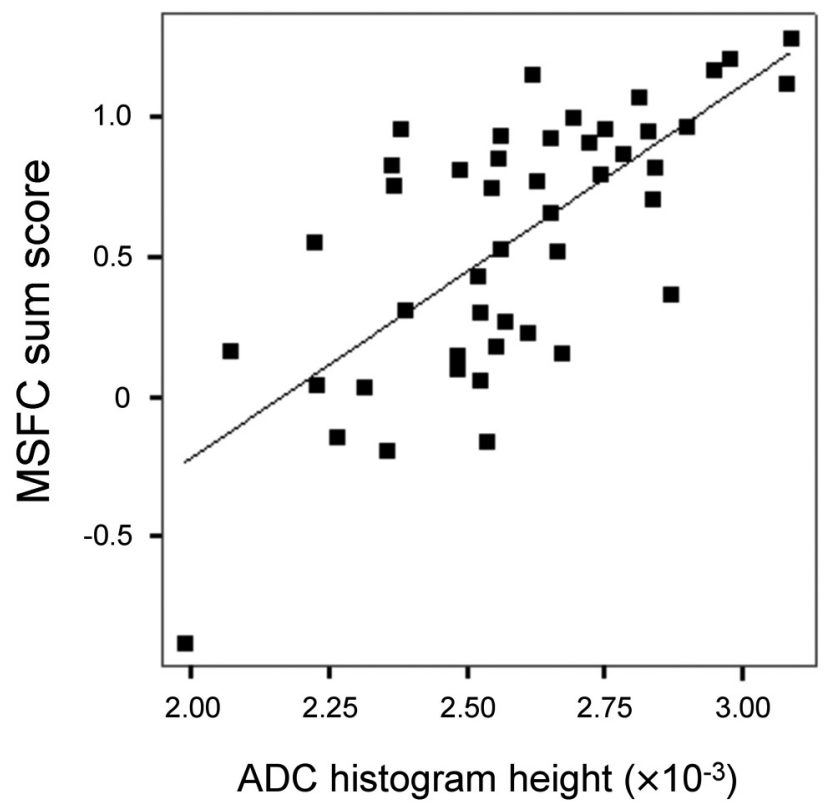

Fig 1. Relationship between ADC histogram peak height and MSFC at follow-up ( $n=46$, $r=0.699$, age-corrected partial $r=0.713, P<.001)$. Patients with acute relapses at the time of assessment were excluded due to potential distortion of their clinical scores.

the gadolinium-enhancement score had no effect on ADC histogram markers or BPF $(P>.05)$.

\section{Interrelation between MRI Markers and Disability}

Interrelations between MRI and disability were highly similar at baseline and follow-up (see On-line Table 2 for details on correlations at follow-up). At follow-up, interrelations with diffusion markers were more distinct $(r=0.759$ [ADC histogram skew, $P<.001]$, Fig 1) than interrelations with WMLL $_{\text {perc }}$ (maximum $r=-0.487$ for MSFC sum score) or BPF (maximum $r=0.438$ for 9 -HPT). EDSS correlated with 4 of 5 ADC histogram markers and with $\mathrm{WMLL}_{\text {perc }}$, but not with BPF.

At baseline, linear regression analysis identified ADC histogram variance as an independent predictor of EDSS $\left(R^{2}=\right.$ $0.137, P=.008)$, MSFC $\left(R^{2}=0.186, P=.003\right)$, and 9 -HPT $\left(R^{2}=0.204, P=.001\right)$. At follow-up, ADC histogram variance was again identified as a predictor of EDSS $\left(R^{2}=0.118, P=\right.$ $.013)$; ADC histogram skew, as a predictor of MSFC $\left(R^{2}=\right.$ $0.533, P<.001)$; and PASAT $\left(R^{2}=0.364, P<.001\right)$ and ADC histogram height, as a predictor of TWT $\left(R^{2}=0.090, P=\right.$ $.032)$ and 9-HPT $\left(R^{2}=0.371, P<.001\right)$.

\section{Prediction of Disease Progression from Baseline MRI Markers}

Among the baseline MRI markers, ADC and BPF allow a differentiation between future MSFC progression versus nonprogression $(P=.042$ and $P=.001$, respectively, Table 4$)$. The latter result was robust toward the Bonferroni correction $(P<$ $.05 / 7 \sim .007$ ) (Fig 2A). The 2 patient subgroups did not differ with regard to age, age at onset, disease duration, baseline MSFC, baseline EDSS, and the proportion of patients with a relapse during the observation interval (24\% and $22 \%$, Fisher exact test, $P=.709)$. Baseline BPF also differentiated between patients with and without EDSS progression $(P=$
Table 4: Baseline MRI and clinical markers of patients with and without MSFC progression

\begin{tabular}{lccc}
\hline & $\begin{array}{c}\text { MSFC } \\
\text { Nonprogression } \\
\text { (Mean) }\end{array}$ & $\begin{array}{c}\text { MSFC } \\
\text { Progression } \\
\text { (Mean) }\end{array}$ & $P$ \\
\hline No. of patients & 25 & 13 & \\
ADC histogram markers & & & \\
$\quad$ Mean $\left(10^{-6} \mathrm{~m}^{2} / \mathrm{s}\right)$ & $0.956 \pm 0.021$ & $0.976 \pm 0.039$ & .042 \\
$\quad$ Variance $\left(10^{-6} \mathrm{~m}^{2} / \mathrm{s}\right)$ & $0.088 \pm 0.008$ & $0.092 \pm 0.008$ & n.s. \\
Skew & $1.035 \pm 0.116$ & $0.951 \pm 0.193$ & n.s. \\
Peak height $\left(10^{-3}\right)$ & $2.662 \pm 0.241$ & $2.539 \pm 0.282$ & n.s. \\
$\quad$ Peak position $\left(10^{-6} \mathrm{~m}^{2} / \mathrm{s}\right)$ & $0.758 \pm 0.017$ & $0.768 \pm 0.018$ & n.s. \\
BPF & $0.828 \pm 0.040$ & $0.783 \pm 0.027$ & $.001^{\mathrm{b}}$ \\
WMLL $(\%)$ & $3.365 \pm 1.673$ & $4.435 \pm 2.228$ & n.s. \\
Clinical variables & & & \\
$\quad$ Age & $36.9 \pm 9.2$ & $39.5 \pm 11.0$ & n.s. \\
$\quad$ Disease duration & $6.6 \pm 6.6$ & $7.5 \pm 7.0$ & n.s. \\
Baseline MSFC & $0.53 \pm 0.46$ & $0.59 \pm 0.33$ & n.s. \\
\hline
\end{tabular}

Note:-n.s. indicates not significant.

${ }^{a}$ Analysis of covariance with 2-level group factor and age as covariate for MRI variables. ${ }^{b} P$ value lower than Bonferroni-corrected $.05 / 7 \sim .007$.

c Two-sided $t$ tests for comparison of clinical variables.

.038). No significant covariate effect of age was found in any comparison.

When we modeled the MSFC subscore changes as continuous variables, mean ADC emerged as an independent predictor of the annual change of $\operatorname{MSFC}\left(F=10.40, R^{2}=\right.$ $0.203, P=.003$, Fig $2 B)$, 9-HPT $\left(F=12.40, R^{2}=0.226, P=\right.$ $.001)$, and PASAT $\left(F=4.36, R^{2}=0.081, P=.044\right)$. For a change of TWT, baseline WMLL $\mathrm{Werc}_{\text {e }}$ emerged as a predictor $\left(F=5.81, P=.021, R^{2}=0.112\right)$.

Post hoc, an effect of intercurrent relapses on these results was excluded as follows: 1) Patients with intercurrent relapses showed a lower (absolute) mean annual MSFC decrease $(n=$ $4,-0.19 \pm 0.11)$ than patients without relapses $(n=9$, $-0.28 \pm 0.26$ ), excluding the finding that MSFC decline was driven by intercurrent relapses. 2) After exclusion of patients with intercurrent relapses, results were stable for the linear regression on the annual MSFC change (baseline mean ADC, $r=0.487, P=.007)$ and for the comparison of baseline BPF between progressive and nonprogressive patients $(P=.0009)$. 3 ) Both results were also stable after restriction to patients with relapsing-remitting MS only $(n=38)$.

\section{Discussion}

Neuroaxonal damage in MS is a strong mediator of clinical impairment ${ }^{1}$ and critical for the development of sustained progression. ${ }^{3}$ This clinico-radiologic study on putative neuroaxonal markers obtained 3 main results: 1) Diffusivity histogram metrics were robust predictors of current disability (MSFC and EDSS). 2) Longitudinal analysis revealed pathologically accelerated changes of diffusivity histogram metrics and whole-brain volume during a median of 12 months, mostly in treated patients with relapsing-remitting MS. 3) Advanced brain atrophy and diffusivity alterations at baseline were associated with MSFC decline, independent of intercurrent relapses. More advanced brain atrophy at baseline was also associated with EDSS decline.

ADC histogram parameters of patients with MS distinctly differed from those of controls, with control values found in the range reported in an earlier normative study. ${ }^{18}$ In MS, 

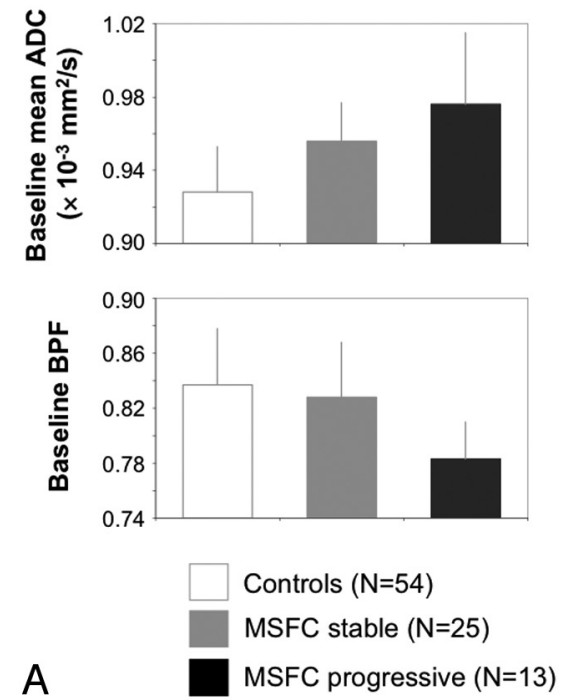

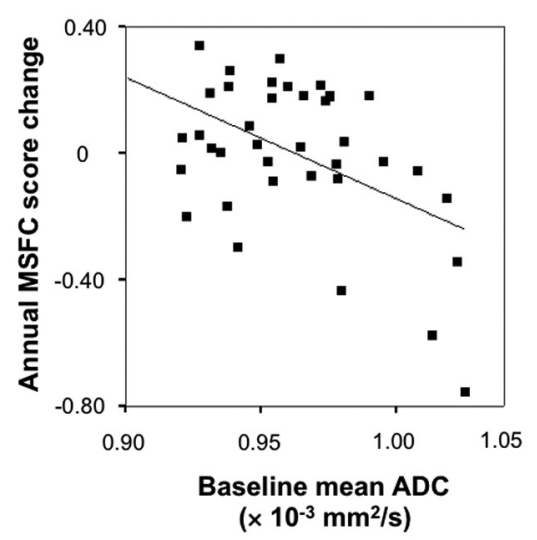

B

Fig 2. Association of MSFC change with baseline mean $A D C$ and BPF. $A$, Bar plots depict baseline mean ADC (upper plot) and BPF (lower plot) for the control group ( $n=54$ ), patients with stable MSFC $(n=25)$, and patients with progressive MSFC $(n=13)$. See "Results" section for statistical details. $B$, Annual MSFC change plotted against baseline mean ADC $(r=$ $-0.473, P=.003)$

abnormal diffusivity is an established finding detectable in focal lesions (ie, WM areas of T2 hyperintensity), whole WM, whole brain, and normal-appearing WM and GM.,18,22,45 Comparisons of ADC values among normal-appearing WM, $\mathrm{T} 1$ isointense, and T1 hypointense lesions suggest that ADC might parallel different degrees of axonal pathology. ${ }^{20,21}$ Similar interrelations between diffusivity and axonal pathology were reported in animal models of MS. ${ }^{19}$ Because axonal injury is considered a key factor of disability in $\mathrm{MS},{ }^{1,3}$ diffusion imaging might serve as a source of particularly useful surrogate markers. In this study, ADC histogram metrics indeed correlated with the patient's current disability status both at baseline and follow-up (see On-line Table 2 for cross-sectional correlations). Mean ADC proved robust across most clinical measures, while even higher correlations were obtained by histogram distribution measures (eg, skew), suggesting that distribution measures detect diffuse or multifocal diffusivity changes most sensitively. ${ }^{16,46}$ As expected, the relationship between the MSFC sum score and $\mathrm{WMLL}_{\text {perc }}$ was weaker, likely because focal T2 hyperintensity is histopathologically unspecific and does not capture diffuse pathology. ${ }^{47}$ Fine motor skills (9-HPT) and cognition (PASAT) also correlated more strongly with diffusivity measures than with BPF and $\mathrm{WMLL}_{\text {perc }}$. Interrelations with ambulatory function were generally weaker, most likely due to the lack of a spinal marker.

Brain volume was the second marker on which we focused. Patients showed a reduced baseline BPF compared with matched controls, as reported by Kalkers et $\mathrm{al}^{6}$ and Rudick et al. ${ }^{48}$ The physiologic negative relationship between BPF and age was disrupted in patients because younger patients already showed a low BPF. Most interesting, younger patients (median, 36.4 years) exhibited a shorter disease duration (4.6 \pm 4.0 versus $9.4 \pm 6.8$ years, $P=.003$ ) compared with the older patients, suggesting that young age at onset could increase the risk of developing brain atrophy. This hypothesis, however, needs support from larger samples. BPF shared only a low proportion of variance with $\mathrm{WMLL}_{\text {perc }}(\sim 22 \%)$, as reported by Guttman et $\mathrm{al}^{49}$ and Simon et al, ${ }^{50}$ confirming that both the pathology of the normal WM and cortical processes contribute substantially to brain atrophy. ${ }^{31,34,51}$ Moderate correlations between BPF and the MSFC sum scores, as described by Kalkers et al, ${ }^{52}$ and with the 9-HPT could be established. No correlation with the PASAT was found, as shown in a previous negative report. ${ }^{53}$ So far, significant BPF/PASAT correlations have only been reported for 82 patients with MS of different clinical subtypes ${ }^{54}$ and for 45 patients with primaryprogressive $\mathrm{MS}^{10}$; however, these patient samples showed a more advanced atrophy.

The longitudinal analysis served to probe whether the proposed markers are suitable to monitor and predict disease progression. In contrast to the clinical ratings, diffusivity measures and BPF showed a significant annual progression that exceeded the values estimated for normal aging. An average annual BPF decrease of approximately $0.4 \%-0.5 \%$ is in line with $0.45 \%$ reported for treated relapsing-remitting $\mathrm{MS},{ }^{33,35}$ larger than the reported rates in healthy subjects $(0.1 \%-$ $0.3 \%),{ }^{4}$ and slightly above $0.36 \%$ observed in patients with relapsing-remitting MS with optimally suppressed inflammatory activity. ${ }^{11}$

Eventually, the longitudinal analysis revealed that more advanced brain volume loss and higher mean ADC at baseline were associated with short-term progression, as primarily defined from the MSFC score. When we modeled this progression as a continuous variable, avoiding arbitrary thresholding, baseline mean ADC emerged as an independent predictor. Changes of the 9-HPT and PASAT subscores that generally contribute strongly to the MSFC score ${ }^{55}$ were also predictable from baseline mean ADC. The same result pattern emerged when the analysis was restricted to patients with relapsingremitting MS and when patients with intercurrent relapses were excluded. Contrary to BPF and diffusivity measures, $\mathrm{WMLL}_{\text {perc }}$ as a focal disease marker showed no progression and proved a weak predictor of current disability and no predictor of disease progression.

While these findings emphasize the impact of nonfocal diffuse pathology, the specificity of the MRI markers for axonal 
pathology cannot be claimed. Postmortem data, for example, have revealed a significant contribution of myelin content to mean diffusivity, ${ }^{56}$ and also in our study, mean ADC and BPF shared approximately $34 \%$ of variance. Diffuse demyelination, through its effects on myelin volume and microscopic diffusion, might therefore influence both volume and ADC measurements. For brain-volume loss in MS, axonal pathology has been proposed to play a prior role because WM volume comprises more axonal (46\%) than myelin volume $(24 \%) .{ }^{4}$ In turn, higher sensitivity of diffusivity toward demyelination is suggested by larger covariation between $\mathrm{WMLL}_{\text {perc }}$ as an indicator of focal demyelination and ADC markers (average $R^{2} \sim 32 \%$ ) compared with BPF $\left(R^{2}=21 \%\right)$. Statistically, results pointed to a higher sensitivity of whole-brain mean ADC compared with BPF in linear prediction models. This effect was robust toward covarying for BPF and remained significant after recalculation of the histograms at a stricter CSF threshold of $1.5 \times 10^{-3} \mathrm{~mm}^{2} / \mathrm{s}$ (data not shown). The correlation between baseline $\mathrm{BPF}$ and disease progression was more robust in that changes of EDSS and MSFC could be predicted, yet it was more nonlinear. Most interesting, such a nonlinear relationship between axonal pathology and secondary progression was hypothesized earlier. ${ }^{3}$

Taken together, we propose that longitudinal results add new evidence for the hypothesis that accumulation of diffuse axonal pathology may (gradually) increase the risk for clinical progression $^{3}$ whereby the contribution of other nonfocal processes, including diffuse demyelination, remains to be clarified. With regard to brain-volume loss, reaching the stage of secondary progression is particularly critical because slowing brain atrophy rates by immunomodulatory therapy then becomes difficult. ${ }^{57}$ Results also demonstrate that subtle clinical progression can be detected by the MSFC score before conventional criteria of secondary disease progression ${ }^{58}$ apply. No predictive value of central atrophy for EDSS progression during 14 months was found in a larger study ${ }^{36}$; however, central atrophy is different from BPF used in this study.

Several limitations of the present study need to be considered. Foremost, the small sample size and the observational design impose limitations on result generalizability. In particular, results are not representative of the spontaneous course of MS because treatment was applied as clinically required. Furthermore, intrinsic to the study design, EDSS or MSFC score progression may reflect a combination of sustained progression as attributable to the natural course of disease and nonresponse to therapy. Technically, higher spatial resolution and fully automated repositioning tools would be preferred, particularly for optimal lesion volumetry. Last, for the generation of ADC histograms, CSF masking was based on a previously reported $^{41}$ fixed ADC threshold, which leads to partial volume effects by macroscopic CSF and hampers a definite attribution of ADC effects to microscopic tissue properties. Indeed, covariation between BPF and mean ADC decreased about linearly when the ADC clipping threshold was lowered. So far, however, it has not been systematically defined which threshold best reflects the true biological correlation between the 2 markers. To categorically avoid partial volume effects, fluid-attenuated DWI may be useful. ${ }^{59}$

\section{Conclusions}

Whole-brain diffusivity and whole-brain volume measurements provide clinically valid and sensitive integral markers to monitor cerebral disease burden in MS during a clinically short time interval of approximately 1 year. The association of advanced brain volume loss and diffusivity changes at baseline with short-term disease progression further suggests that advanced neuroaxonal damage represents a risk for sustained progression.

\section{Acknowledgments}

We thank Reinhold Borschke and Elke Schreiter for their expertise in clinical MRI acquisitions and Rosa Hemauer for supporting image postprocessing. We also gratefully acknowledge Kemal Ates for informatics support and software maintenance; and Emma Bauer, Dr Sandra Lutz, and Dr Felix Müller-Sarnowski for clinical rating. We further thank Dr Erina Schumann-Späth for critical discussion of radiologic aspects of the study and Dorothea Skottke for kind revision of the manuscript.

Disclosures: Susanne Messler—RELATED: Grant. Deutsche Forschungsgemeinschaft, Comments: SFB 386 (Sonderforschungsbereich), ${ }^{*}$ UNRELATED: Employment. Definiens AG, Comments: full-time employee in the field of image analysis in the life sciences area. Frank Weber-UNRELATED: Consultancy. Pfizer-Pharma, Merck-Serono, Orion Pharma; Grants/ Grants Pending. Bayer-Schering AG, ${ }^{*}$ Merck-Serono, ${ }^{*}$ TEVA Pharma GmbH, ${ }^{*}$ Payment for Lectures (including service on speakers bureaus): Bayer-Schering AG, Biogen Idec, Orion Pharma, Comments: honoraria for lectures; Travel/Accommodations/Meeting Expenses Unrelated to Activities Listed: Biogen Idec, Merck-Serono. *Money paid to the institution.

\section{References}

1. Hauser SL, Oksenberg JR. The neurobiology of multiple sclerosis: genes, inflammation, and neurodegeneration. Neuron 2006;52:61-76

2. Anderson VM, Fox NC, Miller DH. Magnetic resonance imaging measures of brain atrophy in multiple sclerosis. J Magn Reson Imaging 2006;23:605-18

3. Trapp BD, Ransohoff R, Rudick R. Axonal pathology in multiple sclerosis: relationship to neurologic disability. Curr Opin Neurol 1999;12:295-302

4. Miller DH, Barkhof F, Frank JA, et al. Measurement of atrophy in multiple sclerosis: pathological basis, methodological aspects and clinical relevance. Brain 2002;125:1676-95

5. Rovaris M, Gass A, Bammer R, et al. Diffusion MRI in multiple sclerosis. Neurology 2005;65:1526-32

6. Kalkers NF, Ameziane N, Bot JC, et al. Longitudinal brain volume measurement in multiple sclerosis: rate of brain atrophy is independent of the disease subtype. Arch Neurol 2002;59:1572-76

7. Chard DT, Griffin CM, Parker GJ, et al. Brain atrophy in clinically early relapsing-remitting multiple sclerosis. Brain 2002;125:327-37

8. De Stefano N, Matthews PM, Filippi M, et al. Evidence of early cortical atrophy in MS: relevance to white matter changes and disability. Neurology 2003;60: 1157-62

9. Zivadinov R, Sepcic J, Nasuelli D, et al. A longitudinal study of brain atrophy and cognitive disturbances in the early phase of relapsing-remitting multiple sclerosis. J Neurol Neurosurg Psychiatry 2001;70:773-80

10. Sastre-Garriga J, Ingle GT, Chard DT, et al. Grey and white matter atrophy in early clinical stages of primary progressive multiple sclerosis. Neuroimage 2004;22:353-59

11. Richert ND, Howard T, Frank JA, et al. Relationship between inflammatory lesions and cerebral atrophy in multiple sclerosis. Neurology 2006;66:551-56

12. De Stefano N, Iannucci G, Sormani MP, et al. MR correlates of cerebral atrophy in patients with multiple sclerosis. J Neurol 2002;249:1072-77

13. Bielekova B, Kadom N, Fisher E, et al. MRI as a marker for disease heterogeneity in multiple sclerosis. Neurology 2005;65:1071-76

14. Fisher E, Rudick RA, Simon JH, et al. Eight-year follow-up study of brain atrophy in patients with MS. Neurology 2002;59:1412-20

15. Horakova D, Dwyer MG, Havrdova E, et al. Gray matter atrophy and disability progression in patients with early relapsing-remitting multiple sclerosis: a 5-year longitudinal study. J Neurol Sci 2009;282:112-19

16. Ciccarelli O, Werring DJ, Wheeler-Kingshott CA, et al. Investigation of MS normal-appearing brain using diffusion tensor MRI with clinical correlations. Neurology 2001;56:926-33

17. Tortorella $\mathrm{P}$, Rocca MA, Mezzapesa DM, et al. MRI quantification of gray and 
white matter damage in patients with early-onset multiple sclerosis. J Neurol 2006;253:903-07

18. Cercignani M, Inglese M, Pagani E, et al. Mean diffusivity and fractional anisotropy histograms of patients with multiple sclerosis. AJNR Am J Neuroradiol 2001;22:952-58

19. Budde MD, Kim JH, Liang HF, et al. Axonal injury detected by in vivo diffusion tensor imaging correlates with neurological disability in a mouse model of multiple sclerosis. NMR Biomed 2008;21:589-97

20. Phuttharak W, Galassi W, Laopaiboon V, et al. ADC measurements in various patterns of multiple sclerosis lesions. J Med Assoc Thai 2006;89:196-204

21. Nusbaum AO, Lu D, Tang CY, et al. Quantitative diffusion measurements in focal multiple sclerosis lesions: correlations with appearance on TI-weighted MR images. AJR Am J Roentgenol 2000;175:821-25

22. Vrenken H, Pouwels PJ, Geurts JJ, et al. Altered diffusion tensor in multiple sclerosis normal-appearing brain tissue: cortical diffusion changes seem related to clinical deterioration. J Magn Reson Imaging 2006;23:628-36

23. Tavazzi E, Dwyer MG, Weinstock-Guttman B, et al. Quantitative diffusion weighted imaging measures in patients with multiple sclerosis. Neuroimage 2007;36:746-54

24. Wilson M, Morgan PS, Lin X, et al. Quantitative diffusion weighted magnetic resonance imaging, cerebral atrophy, and disability in multiple sclerosis. J Neurol Neurosurg Psychiatry 2001;70:318-22

25. Oreja-Guevara C, Rovaris M, Iannucci G, et al. Progressive gray matter damage in patients with relapsing-remitting multiple sclerosis: a longitudinal diffusion tensor magnetic resonance imaging study. Arch Neurol 2005;62:578-84

26. Rovaris M, Judica E, Gallo A, et al. Grey matter damage predicts the evolution of primary progressive multiple sclerosis at 5 years. Brain 2006;129:2628-34

27. Fischer JS, Rudick RA, Cutter GR, et al. The Multiple Sclerosis Functional Composite Measure (MSFC): an integrated approach to MS clinical outcome assessment-National MS Society Clinical Outcomes Assessment Task Force. Mult Scler 1999;5:244-50

28. Cassol E, Ranjeva JP, Ibarrola D, et al. Diffusion tensor imaging in multiple sclerosis: a tool for monitoring changes in normal-appearing white matter. Mult Scler 2004;10:188-96

29. Gallo A, Rovaris M, Riva R, et al. Diffusion-tensor magnetic resonance imaging detects normal-appearing white matter damage unrelated to short-term disease activity in patients at the earliest clinical stage of multiple sclerosis. Arch Neurol 2005;62:803-08

30. Bermel RA, Puli SR, Rudick RA, et al. Prediction of longitudinal brain atrophy in multiple sclerosis by gray matter magnetic resonance imaging $\mathrm{T} 2$ hypointensity. Arch Neurol 2005;62:1371-76

31. Tiberio M, Chard DT, Altmann DR, et al. Gray and white matter volume changes in early RRMS: a 2-year longitudinal study. Neurology 2005;64: 1001-07

32. Chard DT, Griffin CM, Rashid W, et al. Progressive grey matter atrophy in clinically early relapsing-remitting multiple sclerosis. Mult Scler 2004;10: 387-91

33. Paolillo A, Pozzilli C, Giugni E, et al. A 6-year clinical and MRI follow-up study of patients with relapsing-remitting multiple sclerosis treated with interferon-beta. Eur J Neurol 2002;9:645-55

34. Quarantelli M, Ciarmiello A, Morra VB, et al. Brain tissue volume changes in relapsing-remitting multiple sclerosis: correlation with lesion load. Neuroimage 2003;18:360-66

35. Lukas C, Minneboo A, de Groot V, et al. Early central atrophy rate predicts $\mathbf{5}$ year clinical outcome in multiple sclerosis. J Neurol Neurosurg Psychiatry 2010; 81:1351-56. Epub 2010 Sep 8

36. Mesaros S, Rocca MA, Sormani MP, et al. Clinical and conventional MRI predictors of disability and brain atrophy accumulation in RRMS: a large scale, short-term follow-up study. J Neurol 2008;255:1378-83

37. Rovaris M, Gallo A, Valsasina P, et al. Short-term accrual of gray matter pathology in patients with progressive multiple sclerosis: an in vivo study using diffusion tensor MRI. Neuroimage 2005;24:1139-46

38. McDonald WI, Compston A, Edan G, et al. Recommended diagnostic criteria for multiple sclerosis: guidelines from the International Panel on the Diagnosis of Multiple Sclerosis. Ann Neurol 2001;50:121-27

39. Garaci FG, Colangelo V, Ludovici A, et al. A diffusion longitudinal MR imaging study in normal-appearing white matter in untreated relapsing-remitting multiple sclerosis. AJNR Am J Neuroradiol 2007;28:475-78

40. Kurtzke JF. Rating neurological impairment in multiple sclerosis: an expanded disability status scale (EDSS). Neurology 1983;33:1444-52

41. Chabriat H, Pappata S, Poupon C, et al. Clinical severity in CADASIL related to ultrastructural damage in white matter: in vivo study with diffusion tensor MRI. Stroke 1999;30:2637-43

42. Chard DT, Parker GJ, Griffin CM, et al. The reproducibility and sensitivity of brain tissue volume measurements derived from an SPM-based segmentation methodology. J Magn Reson Imaging 2002;15:259-67

43. Smith SM, Zhang Y, Jenkinson M, et al. Accurate, robust, and automated longitudinal and cross-sectional brain change analysis. Neuroimage 2002;17: 479-89

44. Van Leemput K, Maes F, Vandermeulen D, et al. Automated segmentation of multiple sclerosis lesions by model outlier detection. IEEE Trans Med Imaging 2001;20:677-88

45. Cercignani M, Bozzali M, Iannucci G, et al. Magnetisation transfer ratio and mean diffusivity of normal appearing white and grey matter from patients with multiple sclerosis. J Neurol Neurosurg Psychiatry 2001;70:311-17

46. Griffin CM, Chard DT, Ciccarelli O, et al. Diffusion tensor imaging in early relapsing-remitting multiple sclerosis. Mult Scler 2001;7:290-97

47. van Waesberghe JH, Kamphorst W, De Groot CJ, et al. Axonal loss in multiple sclerosis lesions: magnetic resonance imaging insights into substrates of disability. Ann Neurol 1999;46:747-54

48. Rudick RA, Fisher E, Lee JC, et al. Use of the brain parenchymal fraction to measure whole brain atrophy in relapsing-remitting MS. Multiple Sclerosis Collaborative Research Group. Neurology 1999;53:1698-704

49. Guttmann CR, Meier DS, Holland CM. Can MRI reveal phenotypes of multiple sclerosis? Magn Reson Imaging 2006;24:475-81

50. Simon JH, Jacobs LD, Campion MK, et al. A longitudinal study of brain atrophy in relapsing multiple sclerosis: the Multiple Sclerosis Collaborative Research Group (MSCRG). Neurology 1999;53:139-48

51. Kalkers NF, Vrenken H, Uitdehaag BM, et al. Brain atrophy in multiple sclerosis: impact of lesions and of damage of whole brain tissue. Mult Scler 2002;8:410-14

52. Kalkers NF, Bergers E, Castelijns JA, et al. Optimizing the association between disability and biological markers in MS. Neurology 2001;57:1253-58

53. Hildebrandt H, Hahn HK, Kraus JA, et al. Memory performance in multiple sclerosis patients correlates with central brain atrophy. Mult Scler 2006;12: 428-36

54. Lazeron RH, Boringa JB, Schouten $\mathrm{M}$, et al. Brain atrophy and lesion load as explaining parameters for cognitive impairment in multiple sclerosis. Mult Scler 2005;11:524-31

55. Kalkers NF, de Groot V, Lazeron RH, et al. MS functional composite: relation to disease phenotype and disability strata. Neurology 2000;54:1233-39

56. Schmierer K, Altmann DR, Kassim N, et al. Progressive change in primary progressive multiple sclerosis normal-appearing white matter: a serial diffusion magnetic resonance imaging study. Mult Scler 2004;10:182-87

57. Molyneux PD, Kappos L, Polman C, et al. The effect of interferon beta-1b treatment on MRI measures of cerebral atrophy in secondary progressive multiple sclerosis: European Study Group on Interferon Beta-1b in Secondary Progressive Multiple Sclerosis. Brain 2000;123(pt 11):2256-63

58. Rovaris M, Confavreux C, Furlan R, et al. Secondary progressive multiple sclerosis: current knowledge and future challenges. Lancet Neurol 2006;5: 343-54

59. Dichgans M, Putz B, Boos D, et al. Role of subvoxel free fluid on diffusion parameters in brain tissue with cerebral autosomal dominant arteriopathy with subcortical infarcts and leukoencephalopathy and its correlation with physical disability: histogram analysis of standard and fluid-attenuated MR diffusion. AJNR Am I Neuroradiol 2003;24:1083-89 